\title{
OPTIMALITY CONDITIONS FOR NONSMOOTH MULTIOBJECTIVE BILEVEL OPTIMIZATION USING TANGENTIAL SUBDIFFERENTIALS
}

\author{
Mohsine Jennane ${ }^{1}$, El Mostafa Kalmoun ${ }^{2, *}$ (] And Lhoussain El Fadil ${ }^{1}$
}

\begin{abstract}
In combining the value function approach and tangential subdifferentials, we establish necessary optimality conditions of a nonsmooth multiobjective bilevel programming problem under a suitable constraint qualification. The upper level objectives and constraint functions are neither assumed to be necessarily locally Lipschitz nor convex.
\end{abstract}

Mathematics Subject Classification. 49J52, 90C46, 58E35.

Received April 20, 2021. Accepted September 9, 2021.

\section{INTRODUCTION}

Bilevel programming is considered to be one of the important areas in optimization and operations research due to its many applications in various fields (economics, logistics, transportation, engineering and computer science, etc). The problem is stated at two levels of hierarchy in which the feasible set of decisions at the upper level is implicitly related with the solution set at the lower level.

This paper treats the case of bilevel programs in which the upper level decision requires minimizing a vector function $F: \mathbb{R}^{n} \times \mathbb{R}^{m} \rightarrow \mathbb{R}^{p}$, while the lower level involves a single objective $f: \mathbb{R}^{n} \times \mathbb{R}^{m} \rightarrow \mathbb{R}$. This program has the following form

$$
\begin{array}{rl}
\mathbb{R}_{+}^{p}-\min _{x, y} & F(x, y)=\left(F_{1}, \ldots, F_{p}\right)(x, y) \\
\text { subject to } & G_{j}(x, y) \leq 0, \\
& y \in S(x),
\end{array} \quad \text { for all } j \in \mathbb{J}:=\{1, \ldots, q\},
$$

with the upper level constraints being written in terms of the leader's constraint function $G_{j}: \mathbb{R}^{n} \times \mathbb{R}^{m} \rightarrow \mathbb{R}^{q}$, $j \in \mathbb{J}$, as well as the follower's region of rational reactions $S(x)$, which represents the solution set to

$$
\begin{aligned}
& \min _{y} f(x, y) \\
& \text { subject to } g_{i}(x, y) \leq 0, \quad \text { for all } i \in \mathbb{I}:=\{1, \ldots, l\},
\end{aligned}
$$

Keywords. Nonsmooth multiobjective optimization, bilevel programming, optimality conditions, optimal value function, constraint qualifications, tangential subdifferentials.

1 FSDM, Department of Mathematics, Sidi Mohamed Ben Abdellah University, Fez, Morocco.

2 School of Science and Engineering, Al Akhawayn University in Ifrane, PO Box 104, Ifrane 53000, Morocco.

* Corresponding author: E.Kalmoun@aui.ma; kalmoun@gmail.com 
with $g_{i}: \mathbb{R}^{n} \times \mathbb{R}^{m} \rightarrow \mathbb{R}^{l}, i \in \mathbb{I}$, denote the lower level constraints.

The same problem has been investigated by many authors. In employing both the value function as well as the KKT conditions of (1.2), necessary optimality conditions were established in [17] under smooth settings. In [4,9], necessary optimality conditions were derived for Pareto optimal solutions using the Hiriart-Urruty scalarization [7], under the use of Clarke's generalized Jacobian with the nonsmooth Mangasarian-Fromovitz constraint qualification in the first paper [4], and convexificators with a generalized Abadie's constraint qualification in the latter paper [9].

In this work, we take up the general concept of local weakly efficiency with respect to Problem (1.1) which is multiobjective. Letting

$$
\bar{S}:=\left\{(x, y) \in \mathbb{R}^{n} \times \mathbb{R}^{m}: y \in S(x), G_{j}(x, y) \leq 0, \text { for all } j \in \mathbb{J}\right\}
$$

be the leader's feasible region, a pair $(\widehat{x}, \widehat{y}) \in \bar{S}$ is called a local weakly efficient solution of (1.1) and (1.2) if there is an open set $U_{0} \times V_{0}$ containing $(\widehat{x}, \widehat{y})$ such that

$$
F(x, y)-F(\widehat{x}, \widehat{y}) \notin-\operatorname{int} \mathbb{R}_{+}^{n} \quad \forall(x, y) \in \bar{S} \cap\left(U_{0} \times V_{0}\right) .
$$

Our aim is to apply the value function approach as well as the notion of tangential subdifferentials as mathematical tools in order to establish the optimality conditions that must hold at any local weakly efficient solution of the problem (1.1) and (1.2). The latter tool includes many types of subdifferentials like Gâteaux derivatives or convex subdifferentials and coincides with those of Clarke and Michel-Penot, in the case of locally Lipschitz functions that are Clarke regular and MP regular, respectively. It coincides also with upper regular convexificator in the case of tangentially convex functions. Moreover, we employ a specific constraint qualification which is weaker than the Zangwill constraint qualification [19] and other known constraint qualifications like Cottle, Mangasarian-Fromovitz, Kuhn-Tucker, etc.

After this introduction, our paper has the following structure. In Section 2, we give the notations and definitions needed in the sequel. In Section 3, we reformulate the bilevel program (1.1) and (1.2) and we present some constraint qualifications in terms of tangential subdifferentials with a statement of the relationship between them. In Section 4, necessary conditions are derived under a suitable constraint qualification. An example is given at the end to clarify the main result.

\section{NOTATIONS AND PRELIMINARIES}

We follow the standard notation employed in nonlinear optimization. First, letting $\emptyset \neq \mathcal{C} \subseteq \mathbb{R}^{n}$, by the sets $\operatorname{int} \mathcal{C}, \operatorname{co} \mathcal{C}, \mathcal{C}^{\circ}, \mathcal{C}^{s}$ we mean the interior, convex hull, negative and strictly negative polar cone of $\mathcal{C}$, respectively.

The convex cone generated by $\mathcal{C}$ contains exactly all conic combinations of the elements of $\mathcal{C}$, it can be expressed as follows:

$$
\operatorname{cone}(\mathcal{C}):=\left\{\xi \in \mathbb{R}^{n}: \xi=\sum_{i=1}^{r} \alpha_{i} \xi_{i}, \alpha_{i} \geq 0, \xi_{i} \in \mathcal{C}, i=1,2, \ldots, r, r \geq 1\right\}
$$

Recall that for any two sets $\mathcal{C}_{1}$ and $\mathcal{C}_{2}$ in $\mathbb{R}^{n}$ one has

$$
\operatorname{cone}\left(\mathcal{C}_{1} \cup \mathcal{C}_{2}\right)=\operatorname{cone}\left(\mathcal{C}_{1}\right)+\operatorname{cone}\left(\mathcal{C}_{2}\right) .
$$

We recall also three notions of tangent cones: feasible directions, weak feasible directions and contingent, which are given with respect to $\mathcal{C}$ and an element $z \in \operatorname{cl} \mathcal{C}$ as follows

$$
\begin{aligned}
D(\mathcal{C}, z) & :=\left\{\xi \in \mathbb{R}^{n}: \exists \delta>0, \forall \alpha \in(0, \delta), z+\alpha \xi \in \mathcal{C}\right\}, \\
W D(\mathcal{C}, z) & :=\left\{\xi \in \mathbb{R}^{n}: \exists t_{n} \downarrow 0, \forall n \in \mathbb{N}, z+t_{n} \xi \in \mathcal{C}\right\}, \\
T(\mathcal{C}, z) & :=\left\{\xi \in \mathbb{R}^{n}: \exists t_{k} \downarrow 0, \exists \xi_{k} \rightarrow \xi, z+t_{k} \xi_{k} \in \mathcal{C}\right\} .
\end{aligned}
$$


It is a direct matter to check that $D(\mathcal{C}, z) \subseteq W D(\mathcal{C}, z) \subseteq T(\mathcal{C}, z)$. Note that the cone $D(\mathcal{C}, z)$ is in general not convex nor closed, whereas $T(\mathcal{C}, z)$ is closed but not necessarily convex, and when $\mathcal{C}$ is convex, $D(\mathcal{C}, z)=$ $W D(\mathcal{C}, z)$

Let $\varphi: \mathbb{R}^{n} \rightarrow \mathbb{R}$. The function $\varphi$ is said to be tangentially convex at $z \in \mathbb{R}^{n}[15]$ if its directional derivative (also known as Dini derivative) at $z$,

$$
\varphi^{\prime}(z, \xi):=\lim _{t \downarrow 0} \frac{\varphi(z+t \xi)-\varphi(z)}{t}
$$

is finite for any direction $\xi \in \mathbb{R}^{n}$ and convex in this argument. Observe that we have sublinearity of the directional derivative of any tangentially convex function since it is positive homogeneous. Realize that if $\varphi$ is Hadamard directionally differentiable, then its Hadamard directional derivative reduces to it directional derivative. For the converse, $\varphi$ is Hadamard directionally differentiable at $z$ in $\xi$ if $\varphi$ is locally Lipschitz at $z$ and directionally differentiable. On the other hand, the tangential subdifferential of $\varphi: \mathbb{R}^{n} \rightarrow \mathbb{R}$ at $z \in \mathbb{R}^{n}$ is given by $[10,15]$

$$
\partial_{T} \varphi(z):=\left\{y^{*} \in \mathbb{R}^{n}:\left\langle y^{*}, \xi\right\rangle \leq \varphi^{\prime}(z, \xi) \forall \xi \in \mathbb{R}^{n}\right\} .
$$

For a tangentially convex function, this subdifferential is nonempty, compact and convex (see [11]). Furthermore, tangentially convex functions constitute a large class that contains convex functions on open domains where the tangential subdifferential falls into the classical Fréchet subdifferential, Gâteaux differentiable functions on open domains with a tangential subdifferential reduced to the gradient. This class also includes locally Lipschitz functions that are either Clarke regular [1] or Michel-Penot regular [13], and their tangential subdifferential is equal to that of Clarke in the first case and Michel-Penot in the second.

Notice that if $\varphi$ is tangentially convex at $z$, then it follows from the sublinearity of $\varphi^{\prime}(z,$.$) that (2.2)$ is equivalent to

$$
\varphi^{\prime}(z, \xi)=\max _{z^{*} \in \partial_{T} \varphi(z)}\left\langle z^{*}, \xi\right\rangle, \forall \xi \in \mathbb{R}^{n},
$$

which implies that

$$
\inf _{z^{*} \in \partial_{T} \varphi(z)}\left\langle z^{*}, \xi\right\rangle \leq \varphi_{d}^{+}(z, \xi)=\varphi^{\prime}(z, \xi)=\varphi_{d}^{-}(z, \xi) \leq \sup _{z^{*} \in \partial_{T} \varphi(z)}\left\langle z^{*}, \xi\right\rangle, \forall \xi \in \mathbb{R}^{n}
$$

where $\varphi_{d}^{+}(z, \xi)$ and $\varphi_{d}^{-}(z, \xi)$ denote respectively the upper and lower Dini directional derivatives of $\varphi$ at $z$ in the direction $\xi$. Then, $\partial_{T} \varphi(z)$ is a convexificator of $\varphi$ at $z$.

Note also that the definition of tangential subdifferential coincides with that of upper regular convexificator in the case of tangentially convex functions since $\varphi^{\prime}(z, \xi)=\varphi_{d}^{+}(z, \xi)=\sup _{z^{*} \in \partial_{T} \varphi(z)}\left\langle z^{*}, \xi\right\rangle, \forall \xi \in \mathbb{R}^{n}$.

\section{Reformulation of the BILEvel PROGRAM AND CONSTRAint QUALIFICATIONS}

In order to convert the bilevel program (1.1) to an equivalent single-level program, we are going to employ the value function approach $[14,18]$. Let us first denote the value function of the program (1.2) by

$$
\phi(x):=\inf _{y}\left\{f(x, y): g_{i}(x, y) \leq 0 \text { for all } i \in \mathbb{I}\right\}
$$

where by convention $\inf \emptyset=+\infty$. The leader program (1.1) can be restated as follows

$$
\begin{array}{rl}
\mathbb{R}_{+}^{p}-\min _{x, y} & F(x, y)=\left(F_{1}, \ldots, F_{p}\right)(x, y) \\
\text { subject to } & G_{j}(x, y) \leq 0 \\
& g_{i}(x, y) \leq 0
\end{array}
$$

for all $j \in \mathbb{J}$, for all $i \in \mathbb{I}$, 


$$
f(x, y)-\phi(x) \leq 0 .
$$

The feasible set of $(3.2)$ is

$$
\Xi:=\left\{(x, y) \in \mathbb{R}^{n} \times \mathbb{R}^{m} \mid G_{j}(x, y) \leq 0, j \in \mathbb{J}, g_{i}(x, y) \leq 0, i \in \mathbb{I}, f(x, y)-\phi(x) \leq 0\right\} .
$$

Given a point $(\widehat{x}, \widehat{y}) \in \Xi$, we consider the index sets

$$
\mathbb{I}(\widehat{x}, \widehat{y}):=\left\{i \in \mathbb{I}: g_{i}(\widehat{x}, \widehat{y})=0\right\} \quad \text { and } \quad \mathbb{J}(\widehat{x}, \widehat{y}):=\left\{j \in \mathbb{J}: G_{j}(\widehat{x}, \widehat{y})=0\right\} .
$$

Suppose that $F_{k}, k \in \mathbb{K}:=\{1, \ldots, p\}$ and $G_{j}, j \in \mathbb{J}(\widehat{x}, \widehat{y})$ are tangentially convex at $(\widehat{x}, \widehat{y})$, and that $f$ and $g_{i}$, $i \in \mathbb{I}(\widehat{x}, \widehat{y})$ are convex. We can easily show the convexity of $\phi$.

In the following definition, we give three constraint qualifications:

Definition 3.1. Let

$$
\Gamma(\widehat{x}, \widehat{y}):=\bigcup_{i \in \mathbb{I}(\widehat{x}, \widehat{y})} \partial_{T} g_{i}(\widehat{x}, \widehat{y}) \cup \bigcup_{j \in \mathbb{J}(\widehat{x}, \widehat{y})} \partial_{T} G_{j}(\widehat{x}, \widehat{y}) \cup\left(\partial_{T} f(\widehat{x}, \widehat{y})-\partial_{T} \phi(\widehat{x}) \times\{0\}\right) .
$$

We say that:

- Zangwill constraint qualification $\left(\partial_{T}\right.$-ZCQ) is verified at $(\widehat{x}, \widehat{y})$ if

$$
(\Gamma(\widehat{x}, \widehat{y}))^{\circ} \subseteq D(\Xi,(\widehat{x}, \widehat{y})) .
$$

- Weak Zangwill constraint qualification $\left(\partial_{T}\right.$-WZCQ) is verified at $(\widehat{x}, \widehat{y})$ if

$$
(\Gamma(\widehat{x}, \widehat{y}))^{\circ} \subseteq W D(\Xi,(\widehat{x}, \widehat{y})) .
$$

Remark 3.2. Since $D(\Xi,(\widehat{x}, \widehat{y})) \subseteq W D(\Xi,(\widehat{x}, \widehat{y}))$, then $\partial_{T^{-}} \mathrm{ZCQ} \Rightarrow \partial_{T^{-}} \mathrm{WZCQ}$.

\section{NECESSARY OPtimality CONDitions}

Before we proceed to establish our new necessary optimality conditions for local weakly efficient solution of (1.1) by means of the tangential subdifferential, we recall this lemma from [6].

Lemma 4.1 ([6]). Consider two arbitrary index sets $V$ and $W$ (possibly infinite), and two maps $\varphi: V \rightarrow \mathbb{R}^{n}$ and $\psi: W \rightarrow \mathbb{R}^{n}$.

If the set $\operatorname{co}\{\varphi(v), v \in V\}+\operatorname{cone}\{\psi(w), w \in W\}$ is closed, then the following two statements are equivalent:

(i) $\left\{\begin{array}{l}\langle\varphi(v), d\rangle<0, v \in V, V \neq \emptyset, \quad \text { has no solution } d \in \mathbb{R}^{n} . \\ \langle\psi(w), d\rangle \leq 0, w \in W,\end{array}\right.$

(ii) $0 \in \operatorname{co}\{\varphi(v), v \in V\}+\operatorname{cone}\{\psi(w), w \in W\}$.

Theorem 4.2. Assume the point $(\widehat{x}, \widehat{y})$, a local weakly efficient solution of (1.1), is such that

(1) $F_{k}, k \in \mathbb{K}$, and $G_{j}, j \in \mathbb{J}(\widehat{x}, \widehat{y})$ are tangentially convex at $(\widehat{x}, \widehat{y})$, and that $f$ and $g_{i}, i \in \mathbb{I}(\widehat{x}, \widehat{y})$ are convex.

(2) The $\partial_{T}$-WZCQ holds at $(\widehat{x}, \widehat{y})$.

(3) The set

$$
D:=\text { cone }\left(\bigcup_{i \in \mathbb{I}(\widehat{x}, \widehat{y})} \partial_{T} g_{i}(\widehat{x}, \widehat{y}) \cup \bigcup_{j \in \mathbb{J}(\widehat{x}, \widehat{y})} \partial_{T} G_{j}(\widehat{x}, \widehat{y}) \cup\left(\partial_{T} f(\widehat{x}, \widehat{y})-\partial_{T} \phi(\widehat{x}) \times\{0\}\right)\right)
$$

is closed. 
Then there exists $\alpha=\left(\alpha_{1}, \ldots, \alpha_{m}\right) \in \mathbb{R}_{+}^{m}$ with $\sum_{k=1}^{p} \alpha_{k}=1$, such that

$$
\begin{aligned}
0 \in & \sum_{k=1}^{p} \alpha_{i} \partial_{T} F_{k}(\widehat{x}, \widehat{y})+\sum_{i \in \mathbb{I}(\widehat{x}, \widehat{y})} \text { cone } \partial_{T} g_{i}(\widehat{x}, \widehat{y})+\sum_{j \in \mathbb{J}(\widehat{x}, \widehat{y})} \text { cone } \partial_{T} G_{j}(\widehat{x}, \widehat{y}) \\
& +\operatorname{cone}\left(\partial_{T} f(\widehat{x}, \widehat{y})-\partial_{T} \phi(\widehat{x}) \times\{0\}\right) .
\end{aligned}
$$

Proof. First, by definition of local weak efficiency, there is an open set $U \times V$ of $(\widehat{x}, \widehat{y})$ such that there is no $(x, y) \in \Xi \cap(U \times V)$ with

$$
F_{k}(x, y)<F_{k}(\widehat{x}, \widehat{y}) ; \quad \forall k \in \mathbb{K} .
$$

We claim that

$$
\left(\bigcup_{k \in \mathbb{K}} \partial_{T} F_{k}(\widehat{x}, \widehat{y})\right)^{s} \cap W D(\Xi,(\widehat{x}, \widehat{y}))=\emptyset .
$$

Indeed, contrary to our claim, suppose there exists

$$
z^{*}=\left(z_{1}^{*}, z_{2}^{*}\right) \in\left(\bigcup_{k \in \mathbb{K}} \partial_{T} F_{k}(\widehat{x}, \widehat{y})\right)^{s} \cap W D(\Xi,(\widehat{x}, \widehat{y}))
$$

then we get $z^{*} \in\left(\bigcup_{k \in \mathbb{K}} \partial_{T} F_{k}(\widehat{x}, \widehat{y})\right)^{s}$ and $z^{*} \in W D(\Xi,(\widehat{x}, \widehat{y}))$.

Note first that, from $z^{*} \in\left(\bigcup_{k \in \mathbb{K}} \partial_{T} F_{k}(\widehat{x}, \widehat{y})\right)^{s}$, it follows that

$$
\left\langle w^{*}, z^{*}\right\rangle<0, \quad \forall w^{*} \in \partial_{T} F_{k}(\widehat{x}, \widehat{y}), \forall k \in \mathbb{K} .
$$

For each $k \in \mathbb{K}$, define $\varphi_{k}: \partial_{T} F_{k}(\widehat{x}, \widehat{y}) \subset \mathbb{R}^{n} \times \mathbb{R}^{m} \rightarrow \mathbb{R}$ as

$$
\varphi_{k}\left(w^{*}\right)=\left\langle w^{*}, z^{*}\right\rangle, \forall w^{*} \in \partial_{T} F_{k}(\widehat{x}, \widehat{y}) .
$$

The continuity of $\varphi_{k}$ on $\partial_{T} F_{k}(\widehat{x}, \widehat{y})$, which is compact, implies the existence of $\bar{w}^{*} \in \partial_{T} F_{k}(\widehat{x}, \widehat{y})$ with $\varphi_{k}\left(\bar{w}^{*}\right)=$ $\max _{w^{*} \in \partial_{T} F_{k}(\widehat{x}, \widehat{y})}\left\langle w^{*}, z^{*}\right\rangle$. Hence, according to (4.4), we obtain for every $k \in \mathbb{K}$

$$
F_{k}^{\prime}\left(\widehat{x}, \widehat{y}, z^{*}\right)=\max _{w^{*} \in \partial_{T} F_{k}(\widehat{x}, \widehat{y})}\left\langle w^{*}, z^{*}\right\rangle=\left\langle\bar{w}^{*}, z^{*}\right\rangle<0 .
$$

Now, since $z^{*}=\left(z_{1}^{*}, z_{2}^{*}\right) \in W D(\Xi,(\widehat{x}, \widehat{y}))$, there is $t_{s} \downarrow 0$ satisfying $(\widehat{x}, \widehat{y})+t_{s}\left(z_{1}^{*}, z_{2}^{*}\right) \in \Xi$ for all $s \in \mathbb{N}$. Taking into account that the point $(\widehat{x}, \widehat{y})$ is a local weakly efficient solution of (1.1), we obtain that there is $(\widehat{x}, \widehat{y})+t_{s}\left(z_{1}^{*}, z_{2}^{*}\right) \in B((\widehat{x}, \widehat{y}), \delta)$, for some $\delta>0$ and for $s$ large enough, such that there is $\bar{k} \in \mathbb{K}$ satisfying $F_{\bar{k}}\left((\widehat{x}, \widehat{y})+t_{s}\left(z_{1}^{*}, z_{2}^{*}\right)\right) \geq F_{\bar{k}}(\widehat{x}, \widehat{y})$. In combining this with the fact that

$$
F_{\bar{k}}^{\prime}\left((\widehat{x}, \widehat{y}),\left(z_{1}^{*}, z_{2}^{*}\right)\right)=\lim _{s \rightarrow \infty} \frac{F_{\bar{k}}\left((\widehat{x}, \widehat{y})+t_{s}\left(z_{1}^{*}, z_{2}^{*}\right)\right)-F_{\bar{k}}(\widehat{x}, \widehat{y})}{t_{s}},
$$

we obtain $F_{\bar{k}}^{\prime}\left((\widehat{x}, \widehat{y}),\left(z_{1}^{*}, z_{2}^{*}\right)\right) \geq 0$ for $\bar{k} \in \mathbb{K}$, which contradicts (4.5), and consequently, (4.3) is fulfilled. Thus,

$$
\operatorname{int}\left(\bigcup_{k \in \mathbb{K}} \partial_{T} F_{k}(\widehat{x}, \widehat{y})\right)^{s} \cap \operatorname{cl}(W D(\Xi,(\widehat{x}, \widehat{y})))=\left(\bigcup_{k \in \mathbb{K}} \partial_{T} F_{k}(\widehat{x}, \widehat{y})\right)^{s} \cap \operatorname{cl}(W D(\Xi,(\widehat{x}, \widehat{y})))=\emptyset .
$$

On the basis of $\partial_{T^{-}}$WZCQ, we have

$$
\left(\bigcup_{k \in \mathbb{K}} \partial_{T} F_{k}(\widehat{x}, \widehat{y})\right)^{s} \cap\left(\bigcup_{i \in \mathbb{I}(\widehat{x}, \widehat{y})} \partial_{T} g_{i}(\widehat{x}, \widehat{y})\right)^{\circ} \cap\left(\bigcup_{j \in \mathbb{J}(\widehat{x}, \widehat{y})} \partial_{T} G_{j}(\widehat{x}, \widehat{y})\right)^{\circ} \cap\left(\partial_{T} f(\widehat{x}, \widehat{y})-\partial_{T} \phi(\widehat{x}) \times\{0\}\right)^{\circ}=\emptyset .
$$


Then, from (4.6), we see that the system

$$
\begin{cases}\left\langle\zeta_{k}, z^{*}\right\rangle<0, & \forall k \in \mathbb{K}, \forall \zeta_{k} \in \partial_{T} F_{k}(\widehat{x}, \widehat{y}), \\ \left\langle\vartheta_{j}, z^{*}\right\rangle \leq 0, & \forall j \in \mathbb{J}, \forall \vartheta_{j} \in \partial_{T} G_{j}(\widehat{x}, \widehat{y}), \\ \left\langle\theta_{i}, z^{*}\right\rangle \leq 0, & \forall i \in \mathbb{I}, \forall \zeta_{i} \in \partial_{T} g_{i}(\widehat{x}, \widehat{y}), \\ \left\langle\eta, z^{*}\right\rangle \leq 0, & \forall \eta_{k} \in \partial_{T} f(\widehat{x}, \widehat{y})-\partial_{T} \phi(\widehat{x}) \times\{0\}\end{cases}
$$

has no solution $z^{*} \in \mathbb{R}^{n} \times \mathbb{R}^{m}$. On the other hand, since $\partial_{T} F_{k}(\widehat{x}, \widehat{y})$ is compact for each $k \in \mathbb{K}$, the set $\bigcup_{k=1}^{p}\left(\partial_{T} F_{k}(\widehat{x}, \widehat{y})\right)$ is also compact, and hence $\bigcup_{k=1}^{p}\left(\partial_{T} F_{k}(\widehat{x}, \widehat{y})\right)+D$ is closed because so is $D$. Thus, by virtue of Lemma 4.1, we are led to

$$
(0,0) \in \operatorname{co}\left(\bigcup_{k=1}^{p} \partial_{T} F_{k}(\widehat{x}, \widehat{y})\right)+\operatorname{cone}\left(\bigcup_{i \in \mathbb{I}(\widehat{x}, \widehat{y})} \partial_{T} g_{i}(\widehat{x}, \widehat{y}) \cup \bigcup_{j \in \mathbb{J}(\widehat{x}, \widehat{y})} \partial_{T} G_{j}(\widehat{x}, \widehat{y}) \cup\left(\partial_{T} f(\widehat{x}, \widehat{y})-\partial_{T} \phi(\widehat{x}) \times\{0\}\right)\right) .
$$

Taking into account that tangential subdifferentials are convex, there exist nonnegative scalars $\alpha_{k}, k \in \mathbb{K}$ with $\sum_{k=1}^{p} \alpha_{k}=1$ such that

$$
(0,0) \in \sum_{k=1}^{p} \alpha_{k} \partial_{T} F_{k}(\widehat{x}, \widehat{y})+\operatorname{cone}\left(\bigcup_{i \in \mathbb{I}(\widehat{x}, \widehat{y})} \partial_{T} g_{i}(\widehat{x}, \widehat{y}) \cup \bigcup_{j \in \mathbb{J}(\widehat{x}, \widehat{y})} \partial_{T} G_{j}(\widehat{x}, \widehat{y}) \cup\left(\partial_{T} f(\widehat{x}, \widehat{y})-\partial_{T} \phi(\widehat{x}) \times\{0\}\right)\right) .
$$

Consequently by (2.1) that yields

$(0,0) \in \sum_{k=1}^{p} \alpha_{i} \partial_{T} F_{k}(\widehat{x}, \widehat{y})+\sum_{i \in \mathbb{I}(\widehat{x}, \widehat{y})} \operatorname{cone} \partial_{T} g_{i}(\widehat{x}, \widehat{y})+\sum_{j \in \mathbb{J}(\widehat{x}, \widehat{y})} \operatorname{cone} \partial_{T} G_{j}(\widehat{x}, \widehat{y})+\operatorname{cone}\left(\partial_{T} f(\widehat{x}, \widehat{y})-\partial_{T} \phi(\widehat{x}) \times\{0\}\right)$.

Remark 4.3. From Remark 3.2, our main result remains true if we replace $\partial_{T}$-WZCQ with $\partial_{T}$-ZCQ.

Remark 4.4. In (3.2), without the two last inequalities, we obtain the same problem studied in many papers as $[11,12,16]$ with different constraint qualifications. In the future, it is possible to consider finding new results related to bilevel problems based on these studies.

Next, following $[3,8]$ we present an upper estimate for the subdifferential of the value function $\phi$ (which coincides with $\partial_{T} \phi(x)$ since $\phi$ is convex) in terms of the initial data of (1.2).

Proposition 4.5. Suppose that

(i) $f$ is a convex and continuous function.

(ii) The argminimum map $S$ is inner semicontinuous at $(\widehat{x}, \widehat{y})$; that is, for any sequence $\left(x_{k}\right) \in \operatorname{dom} S$ that converges to $\widehat{x}$ there exists a sequence $\left(y_{k}\right) \in S\left(x_{k}\right)$ that converges to $\widehat{y}$.

(iii) $(\widehat{x}, \widehat{y})$ is lower-level regular; that is, the following implication holds

$$
\left[\sum_{i \in \mathbb{I}(\widehat{x}, \widehat{y})} \beta_{i} \widetilde{y}_{i}=0, \beta_{i} \geq 0\right] \Rightarrow\left[\beta_{i}=0 \text { for each } i \in \mathbb{I}(\widehat{x}, \widehat{y})\right]
$$

when $\left(\widetilde{x}_{i}, \widetilde{y}_{i}\right) \in \operatorname{co}\left(\partial_{T} g_{i}(\widehat{x}, \widehat{y})\right)$ with some $\widetilde{x}_{i} \in \mathbb{R}^{n}$ as $i \in \mathbb{I}(\widehat{x}, \widehat{y})$.

Then, an efficient upper estimate of the subdifferential of the value function can be determined as follows:

$$
\partial \phi(\widehat{x}) \subseteq \bigcup_{\left(\theta_{1}, \theta_{2}, \ldots, \theta_{q}\right) \in \Theta(\widehat{x}, \widehat{y})}\left(\partial_{x} f(\widehat{x}, \widehat{y})+\sum_{i \in \mathbb{I}} \theta_{i} \partial_{x} g_{i}(\widehat{x}, \widehat{y})\right)
$$


where

$$
\Theta(\widehat{x}, \widehat{y}):=\left\{\left(\theta_{1}, \theta_{2}, \ldots, \theta_{q}\right) \in \mathbb{R}^{q} \mid 0 \in \partial_{y} f(\widehat{x}, \widehat{y})+\sum_{i \in \mathbb{I}} \theta_{i} \partial_{y} g_{i}(\widehat{x}, \widehat{y}), \theta_{i} \geq 0, \theta_{i} g_{i}(\widehat{x}, \widehat{y})=0, i \in \mathbb{I}\right\} .
$$

Now, we present an example illustrating the main result.

Example 4.6. Consider $n=m=1, p=q=l=2$ and let the leader's objectives and constraints be given by

$$
\begin{aligned}
& F_{1}(x, y)=\left\{\begin{array}{ll}
\frac{x^{3}}{y}-x, & y \neq 0, \\
-x, & y=0,
\end{array} \quad F_{2}(x, y)=\max \{x, y\}\right. \\
& G_{1}(x, y)=-x, \quad G_{2}(x, y)=x^{3}-4 x .
\end{aligned}
$$

The follower's objective and constraints are as follows

$$
f(x, y)=|x|+|y|, \quad g_{1}(x, y)=y-1 \quad g_{2}(x, y)=-y .
$$

Then, after straightforward calculations, one has $\Xi=[0,2] \times\{0\}$. The convexity of $f$ and $g_{1}$ can be easily checked as well as the tangential convexity of $F_{1}, G_{1}$ and $G_{2}$ at the local weakly efficient point $(\widehat{x}, \widehat{y}):=(0,0)$.

In this example, the follower's region of rational reactions $S$ and the function $\phi$ are

$$
S(x)=\{0\}, \quad \phi(x)=|x| .
$$

Let us show that $\mathbb{I}(\widehat{x}, \widehat{y})=\{2\}$ and $\mathbb{J}(\widehat{x}, \widehat{y})=\{1,2\}$. Moreover, the tangential subdifferentials of $F_{1}, F_{2}, f, \phi$, $G_{1}, G_{2}$ and $g_{2}$, are given by

$$
\begin{aligned}
\partial_{T} F_{1}(\widehat{x}, \widehat{y}) & =\{(-1,0)\}, \quad \partial_{T} F_{2}(\widehat{x}, \widehat{y})=\operatorname{co}\{(0,1) ;(1,0)\} \\
\partial_{T} f(\widehat{x}, \widehat{y}) & =[-1,1] \times[-1,1], \quad \partial_{T} \phi(\widehat{x})=[-1,1], \\
\partial_{T} G_{1}(\widehat{x}, \widehat{y}) & =\{(-1,0)\}, \quad \partial_{T} G_{2}(\widehat{x}, \widehat{y})=\{(-4,0)\}, \quad \partial_{T} g_{2}(\widehat{x}, \widehat{y})=\{(0,-1)\} .
\end{aligned}
$$

The weak feasible directions cone w.r.t. $\Xi$ and $(\widehat{x}, \widehat{y})$ is given as follows $\Xi=W D(\Xi,(\widehat{x}, \widehat{y}))=\mathbb{R}^{+} \times\{0\}$. A simple calculation can lead to

$$
(\Gamma(\widehat{x}, \widehat{y}))^{\circ} \subseteq W D(\Xi,(\widehat{x}, \widehat{y})),
$$

which mean our constraint qualification $\partial_{T}$-WZCQ holds at $(\widehat{x}, \widehat{y})$.

We conclude that all assumptions of Theorem 4.2 are fulfilled. Taking $\alpha_{1}=\alpha_{2}=\frac{1}{2}$, we can easily verify the optimality condition given in this theorem:

$$
\begin{aligned}
(0,0) \in & \alpha_{1} \partial_{T} F_{1}(\widehat{x}, \widehat{y})+\alpha_{2} \partial_{T} F_{2}(\widehat{x}, \widehat{y})+\text { cone } \partial_{T} G_{1}(\widehat{x}, \widehat{y})+\text { cone } \partial_{T} G_{2}(\widehat{x}, \widehat{y}) \\
& + \text { cone } \partial_{T} g_{2}(\widehat{x}, \widehat{y})+\operatorname{cone}\left(\partial_{T} f(\widehat{x}, \widehat{y})-\partial_{T} \phi(\widehat{x}) \times\{0\}\right)
\end{aligned}
$$

In the above example, we can obtain a similar result if we have only $F=F_{1}$. In this case, it's worth noting that Theorem 5.1 of [8] and Theorem 1 of [5] cannot be employed because $F_{1}$ is not locally Lipschitz at the local weakly efficient $(\widehat{x}, \widehat{y})=(0,0)$. It follows that our main result is more general since the leader's objective can be multiobjective and not necessarily locally Lipschitz. On the other hand, since the functions $F_{1}$ and $G_{2}$ are not convex, we cannot apply the results that require the convexity of the functions in the upper level such as Theorem 4.1 of [2].

Acknowledgements. We would like to express our sincere thanks to the two anonymous reviewers referees for their helpful comments and suggestions. 


\section{REFERENCES}

[1] F.H. Clarke, Optimization and Nonsmooth Analysis. Wiley, New York (1983).

[2] S. Dempe, J. Dutta and B.S. Mordukhovich, New necessary optimality conditions in optimistic bilevel programming. Optimization 56 (2007) 577-604.

[3] N.A. Gadhi, A note on the paper "Sufficient optimality conditions using convexifactors for optimistic bilevel programming problem". J. Ind. Manag. Optim. (2021) DOI: 10.3934/jimo.2021103.

[4] N. Gadhi and S. Dempe, Necessary optimality conditions and a new approach to multi-objective bilevel optimization problems. J. Optim. Theory Appl. 155 (2012) 100-114.

[5] N.A. Gadhi and L. Lafhim, Optimality conditions for a bilevel optimization problem in terms of KKT multipliers and convexificators. Croatian Oper. Res. Rev. 10 (2019) 329-335.

[6] M.A. Goberna and M.A. López, Linear Semi-Infinite Optimization. Wiley, Chichester (1998).

[7] J.B. Hiriart-Urruty, Tangent cones, generalized gradients and mathematical programming in Banach spaces. Math. Oper. Res. 4 (1979) 79-97.

[8] B. Kohli, Optimality conditions for optimistic bilevel programming problem using convexifactors. J. Optim. Theory Appl. 152 (2012) 632-651.

[9] L. Lafhim, N. Gadhi, K. Hamdaoui and F. Rahou, Necessary optimality conditions for a bilevel multiobjective programming problem via a $\Psi$-reformulation. Optimization 67 (2018) 2179-2189.

[10] C. Lemaréchal, An introduction to the theory of nonsmooth optimization. Optimization 17 (1986) 827-858.

[11] J.E. Martínez-Legaz, Optimality conditions for pseudoconvex minimization over convex sets defined by tangentially convex constraints. Optim. Lett. 9 (2015) 1017-1023.

[12] F. Mashkoorzadeh, N. Movahedian and S. Nobakhtian, Optimality conditions for nonconvex constrained optimization problems. Num. Funct. Anal. Optim. 40 (2019) 1918-1938.

[13] P. Michel and J.P. Penot, A generalized derivative for calm and stable functions. Diff. Int. Equ. 5 (1992) $433-454$.

[14] J.V. Outrata, On the numerical solution of a class of Stackelberg problems. ZOR-Methods Mod. Oper. Res. 34 (1990) $255-277$.

[15] B.N. Pshenichnyi, Necessary Conditions for an Extremum. Marcel Dekker Inc., New York, NY (1971).

[16] N. Sisarat and R. Wangkeeree, Characterizing the solution set of convex optimization problems without convexity of constraints. Optim. Lett. 14 (2020) 1127-1144.

[17] J.J. Ye, Necessary optimality conditions for multiobjective bilevel programs. Math. Oper. Res. 36 (2011) $165-184$.

[18] J.J. Ye and D.L Zhu, Optimality conditions for bilevel programming problems. Optimization 33 (1995) 9-27.

[19] W.I. Zangwill, Nonlinear Programming: A Unified Approach. Prentice-Hall, Englewood Cliffs, NJ (1969).

\section{Subscribe to Open (S2O) A fair and sustainable open access model}

This journal is currently published in open access under a Subscribe-to-Open model (S2O). S2O is a transformative model that aims to move subscription journals to open access. Open access is the free, immediate, online availability of research articles combined with the rights to use these articles fully in the digital environment. We are thankful to our subscribers and sponsors for making it possible to publish this journal in open access, free of charge for authors.

\section{Please help to maintain this journal in open access!}

Check that your library subscribes to the journal, or make a personal donation to the S2O programme, by contacting subscribers@edpsciences.org

More information, including a list of sponsors and a financial transparency report, available at: https://www. edpsciences.org/en/maths-s2o-programme 